\title{
Deciphering cellular and molecular causes of the tumor functional heterogeneity of liver cancer
}

\author{
Experimental \& Molecular Medicine (2018) 50, e415; doi:10.1038/emm.2017.188; published online 5 January 2018
}

$\mathrm{L}$ iver cancer is one of the most common and deadly cancers in the world with an annual incidence of $0.25-1$ million cases; $\sim 90 \%$ of those cases are hepatocellular carcinoma (HCC). Several therapeutic options exist for treating HCC, including surgical resection, liver transplantation, radiofrequency ablation, transarterial chemoembolization and systemic chemotherapy (using drugs such as sorafenib). However, only 10-20\% of patients with HCC are currently eligible for surgical intervention. Moreover, patients undergoing resection often have a high frequency of recurrence, and postoperative 5 -year survival is only $30-40 \%$. Thus, one challenge of HCC treatment is the frequent recurrence after curative resection. Early recurrence occurs within 2 years after resection, whereas late recurrence mainly results from de novo or independent liver tumors as a consequence of the strong carcinogenic background. Recurrent or multifocal liver tumors are considered to be originated from a primary tumor via two mechanisms: multi-centric occurrence and intrahepatic metastasis.

There is increasing recognition and appreciation that tumors comprise complex networks that resemble an intricate ecosystem, and individual cells endowed with distinctive characteristics play different functional roles and cooperate to support the growth and maintenance of the whole tumor. Driver mutations and heritable epigenetic changes give rise to different clones of tumor cells during the course of tumor formation, progression and metastasis. Molecular heterogeneity in HCC has two configurations. One relates to the distinction between multi-centric occurrences vs. intrahepatic metastasis in patients with multinodular disease. This is almost unique to HCC because of its frequent development in the background of chronic liver disease. The discrimination between these two types of multiple liver tumors is clinically crucial because each type exhibits biologically different behaviors. The second configuration of HCC heterogeneity refers to intratumor molecular diversity, which has been extensively studied in other solid tumors, and there are very few reports on intratumor molecular heterogeneity in HCC. Thus, there is still considerable uncertainty about the extent and consequences of molecular heterogeneity in HCC.
Although HCC is caused by clear etiologies and some studies have proposed classifying HCC based on phenotypes, molecular changes or pathway activation, no driver events corresponding to targeted therapy have been identified. Thus, detection of HCC intratumor heterogeneity is important for the development of effective targeted therapies. Although liver transplantation, surgical resection and radiofrequency ablation offer a curative treatment for HCC, it is not an option for patients with intermediate/advanced stage HCC. Thus, a better understanding of the intratumor heterogeneity of HCC should provide critical knowledge about the prognosis of the disease and response to potential future therapy. In this special issue, we invited one review and seven original articles, discussed recent findings on the molecular and cellular heterogeneity of HCC and highlighted new findings from functional studies on the development of novel therapeutic targets for treating HCC.

Xin Wei Wang's group (NCI, NIH, USA) reviewed the significance of intertumor and intratumor heterogeneity in liver cancer. Tumor heterogeneity consists of intertumor (tumor by tumor) and intratumor (within a tumor) heterogeneity. Intertumor heterogeneity refers to primary liver cancers from different patients whose altered genotype and phenotype are induced by diverse etiological and environmental factors. By contrast, intratumor heterogeneity refers to genomic and biological variations within a tumor lesion gained by tumor cell evolution under diverse microenvironments linked to different etiologies. Here they proposed a concept of intratumor 'functional' heterogeneity, which describes the ability of cancer cells to undergo cellular proliferation, adaptation and drug resistance within a defined microenvironment linked to a specific etiology. They also discussed approaches to study intratumor heterogeneity and suggested that improving the assessment of the complexity of primary liver cancer intratumor heterogeneity will provide a better understanding of functional heterogeneity, which may be linked to therapeutic responses.

Suk Woo Nam's group (The Catholic Univ. of Korea, Korea) investigated the early metastasis enhancing gene TCIRG1 (T-cell immune regulator 1) and showed that TCIRG1 is a 
determining factor for early recurrence of HCC after surgical resection of the primary lesion. TCIRG1 is upregulated in HCC, and its targeted inactivation inhibits liver tumorigenesis in vitro and in vivo by selectively modulating EMT-related proteins in HCC cells. This finding indicates that the development of specific modulators of TCIRG1 may be crucial to controlling tumor progression or even the recurrence of HCC after resection. Laura Beretta's group (MD Anderson Cancer Center, USA) showed that S100A4+ cells do not contribute to HCC onset but maintain the stemness phenotype of the tumor. They generated a new mouse model of HCC with stemness properties, allowing for the depletion of S100A4+ cells by crossing mice with a hepatic deletion of phosphatase and tensin homolog (PTEN) with mice expressing viral thymidine kinase under the control of the S100A4 promoter. They showed that although depleting S100A4+ cells did not prevent the development of HCC, it reduced the stemness of the tumor as measured by the expression of progenitor cell markers CD24 and osteopontin and biliary marker KRT19. These results suggest crosstalk between inflammation and stemness. Xin Chen's group (UCSF, USA) showed that the loss of PTEN synergizes with overexpression of c-Met and promotes HCC development in mice (sgPten/c-Met). Using Rictor conditional knockout mice, they demonstrated that sgPten/c-Met-driven HCC development strictly depends on an intact mTORC2 complex supporting the critical role of mTORC2 in hepatocarcinogenesis.

Yujin Hoshida's group (Icahn School of Medicine at Mount Sinai, USA) focused on the tractable experimental model that accounts for intertumor molecular heterogeneity. In this study, they provided comprehensive reference information for a panel of widely used and commercially available hepatoma cell lines as in vitro models of human HCC molecular subtypes and proposed that hepatoma cell lines with designated HCC subtypes represent an invaluable resource as experimental models that retain intratumor/intertumor/patient heterogeneity for anticancer drug assessment and development. Ju-Seog Lee's group (MD Anderson Cancer Center, USA) highlighted the importance of glutamine metabolism in the regulation of autophagy in HCC cells. In-Sun Chu's group (KRIBB, Korea) examined an association between the gene expression signature of FOXM1 and HCC patient outcomes. In a multivariate analysis, the coexpressed gene set of FOXM1 was the most significant prognostic factor for overall survival in patients with HCC. In addition, they verified the association between the coexpressed gene set of FOXM1 and patient prognosis in different types of cancer, including pancreatic adenocarcinoma, lung adenocarcinoma, breast carcinoma and bladder urothelial carcinoma. Lastly, Randy Johnson's group (MD Anderson Cancer Center, USA) demonstrated that the Hippo signaling pathway coactivators, Yap and Taz, are not essential for achieving a proper liver size during development or in the perinatal period but are required to mount an effective regenerative response following partial hepatectomy.

\section{CONFLICT OF INTEREST}

The author declares no conflict of interest.

Suk Woo Nam ${ }^{1,2}$

${ }^{1}$ Department of Pathology, College of Medicine, The Catholic

University of Korea, Seocho-gu, Seoul, Republic of Korea and

${ }^{2}$ Functional RNomics Research Center, The Catholic University of Korea, Seocho-gu, Seoul, Republic of Korea E-mail:swnam@catholic.ac.kr

This work is licensed under a Creative Commons Attribution-NonCommercial-NoDerivs 4.0 International License. The images or other third party material in this article are included in the article's Creative Commons license, unless indicated otherwise in the credit line; if the material is not included under the Creative Commons license, users will need to obtain permission from the license holder to reproduce the material. To view a copy of this license, visit http://creativecommons.org/licenses/by-nc-nd/4.0/

(C) The Author(s) 2018 